\title{
CONCEPTO DE COMPETENCIAS EN MAESTROS DEL ÁREA DE LENGUA CASTELLANA EN BOGOTÁ ${ }^{1}$
}

\author{
JULIO ALEXÁNDER BERNAL CHÁVEZ* \\ GREYSI MILENA GIRALDO AGUIRRE* *
}

\section{Resumen}

Este documento determina la incidencia de la teoría de las competencias lingüísticas y comunicativas en las justificaciones presentadas por los maestros e investigadores de lengua castellana en relación con su uso en el contexto educativo. Se traza como metas específicas desglosar las teorías que fundamentan y justifican el concepto de competencia, determinar la recontextualización que algunos investigadores hacen respecto a las competencias e identificar las justificaciones de los maestros e investigadores de lengua castellana acerca de las competencias.

Palabras clave: educación, competencia lingüística, competencia comunicativa.

\section{Abstract}

The present document determines the incidence of the theory of linguistic and communicative competences in the justifications presented by the teachers and researchers of Spanish Language Arts classes with respect with their use in the educational context. As specific targets chosen we find to break down or analyze the theories that fundament and justify the concept of competence, to determine the recontextualization (second interweaving) that some researchers make with respect to the competences and identify the justifications of the teachers of Spanish language Arts classes and researchers of Spanish language about the competences.

Keywords: education, linguistic competence, communicative competence
}

\section{INTRODUCCIÓN}

Desde la década de los ochenta, en Colombia se ha introducido el concepto de competencia comunicativa en la enseñanza de la lengua castellana, proveniente de la teoría del antropólogo Dell Hymes quien, a su vez, la retoma de la teoría de la competencia lingüística de Noam Chomsky.

Desde esta perspectiva, el actual artículo pretende determinar la incidencia del concepto de competencias en el área de enseñanza de lengua castellana en Colombia. Para tal efecto se enunciará el recorrido del concepto desde Chomsky, pasando por los investigadores colombianos que trabajan el tema, para llegar, finalmente; a los conceptos presentados por los maestros en Bogotá. De esta manera, se expondrán, en primer lugar, aproximaciones teóricas del concepto de competencia desde Noam Chomsky, Dell Hymes, y algunos investigadores en Colombia; en segundo lugar, se presentan los resultados de un análisis de contenido hecho a 17 entrevistas realizadas a maestros

\footnotetext{
${ }^{1}$ El siguiente artículo hace parte de los resultados de la investigación, dirigida por Marieta Quintero, titulada "De la competencia lingüística a la competencia comunicativa y su incidencia en contextos educativos", realizada como monografía para acceder al título de licenciados en lingüística y literatura otorgado por la Universidad Distrital Francisco José de Caldas, en diciembre de 2003.

* Licenciado en lingüística y literatura de la Universidad Distrital Francisco José de Caldas. Estudiante de maestría, Instituto Caro y Cuervo. Correo electrónico: juliobernal763@hotmail.com

** Licenciada en lingüística y literatura de la Universidad Distrital Francisco José de Caldas.
}

ARTículo RECIBIDo 30 DE MAYO DE 2004. ACEPTADo 13 DE JUNIO DE 2004. 
investigadores de educación básica, media y superior, a partir del cual se identifica el concepto de competencia que tienen los maestros de lengua castellana en Bogotá.

\section{COMPETENCIA LINGÜÍSTICA}

El concepto de competencia lingüística fue desarrollado por Noam Chomsky, cuyo interés central, en toda su obra, ha sido profundizar en el conocimiento de la naturaleza del lenguaje y de los procesos y estructuras mentales que fundamentan el uso y adquisición del mismo. Chomsky, concentra su atención en la lingüística cartesiana que parte de los postulados teóricos de Renato Descartes en el siglo XVII, en los cuales se formula con claridad el interrogante acerca de la capacidad humana de comprender y la naturaleza de la inteligencia. Tal preocupación fue retomada en el siglo XVII por la escuela de Port Royal y Cordemoy, y en el siglo XVIII por Herder y Humboldt, entre otros.

El término de competencia lingüística encuentra sus antecedentes tanto en la capacidad creativa del lenguaje humano descrita por Descartes $(1975)^{2}$, como en los postulados de Humboldt, quien propone el lenguaje como energeia o movimiento dado que, con un número finito de reglas, puede generar o crear un número infinito de oraciones.

Chomsky considera que la lengua está constituida por una estructura subyacente y una superficial, que por medio de unas reglas de transformación generan todas las oraciones posibles, tal proceso explica el aspecto creativo del uso lingüístico (Chomsky, 1970). En esta vía, Chomsky propone la teoría de la gramática generativa transformacional, mediante la cual formula leyes gramáticas generales; para ello, diseña elementos metodológicos de justificación y adecuación de la competencia de un hablante-oyente y su conocimiento de la lengua.

Sin embargo, esta competencia no puede ser observada directamente, ni obtenida de los datos por procedimientos inductivos ya que para determinar unas reglas de transformación gramatical es necesario desarrollar una metodología que trabaje con un hablante-oyente ideal (Chomsky, 1970) a partir de la estructura de la oración.

Con la preocupación lingüística por un hablante-oyente ideal en condiciones ideales, surge en Chomsky la pregunta acerca de la distinción fun- damental entre competencia entendida como el conocimiento que el hablante-oyente ideal tiene de su lengua y actuación como el uso real de la lengua en situaciones concretas. Se aclara que Chomsky concibe la actuación como el reflejo directo de la competencia solamente a partir de una idealización (Chomsky, 1970).

Cuando Chomsky determina, por un lado, al hablante oyente ideal como preocupación central de la teoría lingüística y, por otro lado, la actuación como un acto abstracto, está usando una metodología que le ayude a construir una representación de la facultad del lenguaje en una gramática. Todo esto, con el fin de reconocer "el estado cognitivo alcanzado en el aprendizaje lingüístico" $(1970)^{3}$. De esta manera, Chomsky expone que la competencia refleja los procesos de mente humana (Chomsky, 1979) ${ }^{4}$, que dan cuenta de la estructura y funcionamiento del lenguaje.

\section{2. $L A$ COMPETENCIA COMUNICATIVA}

En oposición a la propuesta Chomskiana, aparecen, en 1968, los planteamientos del teórico Dell Hymes quien propone la teoría de la competencia comunicativa desde condiciones socioculturales.

Dell Hymes, a diferencia de la idealización Chomskiana de un hablante-oyente ideal caracterizado por pertenecer a una comunidad lingüística homogénea, al que no lo afectan condiciones, como limitaciones de memoria, distracciones, cambios de centros de atención e interés, ni errores al aplicar su conocimiento de la lengua al uso real (Chomsky, 1970), propone un hablante-oyente real ubicado en un contexto sociocultural determinado, que se ve afectado por situaciones históricoculturales particulares y por condiciones del medio ambiente.

En este sentido, Hymes, encuentra problemático el concepto de hablante-oyente ideal propuesto en la competencia lingüística. Hymes muestra

\footnotetext{
2 “[...] es una cosa bien notable que no haya hombres [...] que no sean capaces de ordenar un conjunto de diversas palabras con el que den a conocer sus pensamientos", p. 160.

3 "Un hablante-oyente ideal, en una comunidad lingüística del todo homogénea, que sabe su lengua perfectamente ya que no lo afectan condiciones sin valor gramatical, como son limitaciones de memoria, distracciones, cambios de centro de atención e interés y errores al aplicar su conocimiento de la lengua al uso real".

${ }^{4}$ En tal sentido "la teoría del lenguaje es simplemente esa parte de la psicología general que se ocupa de un órgano mental particular: el lenguaje humano", p. 50.
} 
que la teoría Chomskiana no proporciona elementos de tipo teórico y epistemológico que permitan dar soluciones a problemas sociolingüísticos: "el enfrentamiento con la realidad de los niños como seres que se comunican requieren una teoría dentro de la cual los factores socioculturales jueguen un papel explicito constitutivo; factores éstos que no han sido considerados" (Hymes, 1996, p. 15).

Hymes, encamina sus estudios hacia la explicación de situaciones socioculturales que involucran factores del medio ambiente, motivaciones personales, valores culturales, enfermedades y otra serie de variables.

Aunque Hymes incluye factores cognoscitivos en la competencia comunicativa, otorga mayor importancia a las motivaciones que propician los actos de habla, con lo cual se evidencia la prioridad que en la competencia comunicativa tienen variables provenientes de la interacción sociocultural (Hymes, 1996) ${ }^{5}$.

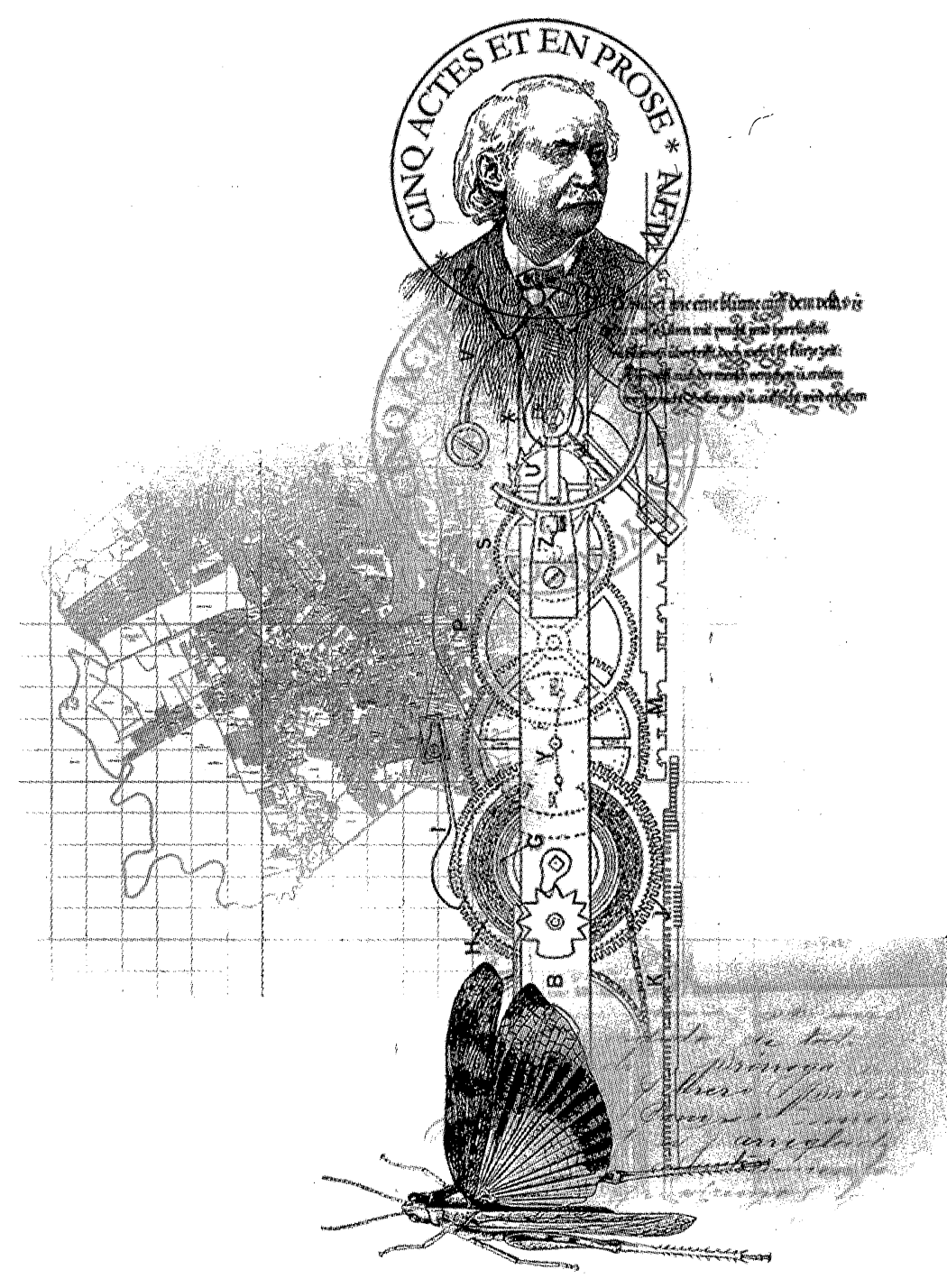

En consecuencia, ante la propuesta de la competencia lingüística de Chomsky, aparece la teoría de la competencia comunicativa. Hymes, afirma que la ciencia lingüística debe tener un enfoque bidimensional que no sólo proporcione una explicación de la estructura gramatical, como lo hacían todas las teorías lingüísticas, sino que establezca una relación entre dichas teorías con los sistema reales de uso, con el fin dar relevancia a los elementos socioculturales que inciden en los actos de habla.

Mientras que para Chomsky el aspecto comunicativo de la lengua no es interesante, para Hymes se constituye en el objeto principal de estudio. En consecuencia, Hymes argumenta que la lingüística se ha limitado a estudios estructurales ubicados dentro de un campo puramente teórico, que solamente llegará a ser completa cuando se realicen análisis de los actos de habla teniendo en cuenta los contextos específicos y las situaciones particulares.

En la misma vía, Hymes considera que se debe superar la delimitación Chomskiana entre competencia y actuación, aunque reconoce que para Chomsky la actuación es una abstracción que refleja la competencia en situaciones ideales, considera que no se puede prescindir del acto social en el cual se hace uso de la competencia lingüística.

Así mismo, Hymes establece la pertinencia como criterio esencial para la teoría de la competencia comunicativa. Esta pertinencia consiste en la respuesta que el hablante-oyente da en la comunicación dependiendo del contexto. En tal sentido, un acto comunicativo se define a partir de la capacidad que tienen los hablantes-oyentes para reconocer los factores socioculturales apropiados que permitan su actuación.

Además de la competencia lingüística, ubicada en una estructura profunda, Hymes, propone una competencia para el uso, ubicada en una estructura superficial que refleje la actuación real. Por lo tanto, a diferencia de Chomsky, Hymes considera que el medio sociocultural influye en la conformación tanto de la estructura superficial como de la estructura subyacente: "la actuación (competencia comunicativa) es real y la competencia (lingüística) es subyacente" (1996, p. 25).

\footnotetext{
5 "La especificación de la habilidad para el uso como parte de la competencia permite la participación de factores no cognoscitivos, tales como la motivación, en la determinación de la competencia", p. 27.
} 
De esta manera, la competencia desde la propuesta de Hymes, es una capacidad subyacente, que adquiere valor cultural al momento de ser usada. El uso del lenguaje responde a condicionamientos socioculturales que determinan los juicios y las habilidades de los usuarios para realizar actos de habla.

En tal sentido, para Hymes la competencia comunicativa es una habilidad para el uso de la lengua que depende tanto de un conocimiento tácito de las reglas de formación gramatical, como de un conocimiento tácito de reglas culturales que delimitan la posibilidad que un hablanteoyente real tiene para usar comunicativamente la lengua.

Además de la delimitación entre una estructura profunda y una estructura superficial en la competencia comunicativa, Hymes, reconoce el aspecto creativo del uso del lenguaje, no solamente como la capacidad para generar un número infinito de oraciones, sino como la posibilidad del usuario, tanto para idearse oraciones nuevas apropiadas a las situaciones, como para entender nuevas oraciones producidas por otros.

En conclusión, Dell Hymes se opone en varios aspectos a la teoría de la competencia lingüística. En primer lugar, critica la postura meramente lingüística, argumentando que Chomsky descuida los aspectos socioculturales. En tal sentido, ante la idealización lingüística de un hablante-oyente ideal, Hymes propone un hablante oyente real. En segundo lugar, aunque Hymes reconoce la competencia lingüística como estructura profunda y la actuación como estructura superficial, considera que también existe una competencia de uso conformada tanto por estructuras intrínsecas socioculturales, como por la habilidad para comunicarse. En tercer lugar, Hymes señala que, al mismo tiempo que existe un aspecto creativo gramatical para producir oraciones, aparece un aspecto creativo de uso que permite al hablante-oyente real idearse y comprender oraciones en lenguaje natural.

\section{ALGUNOS CONCEPTOS DE COMPETENCIA QUE CIRCULAN EN COLOMBIA}

En Colombia la preocupación por el estudio de las competencias surge en los años ochenta con estudiosos e investigadores como Nicolás Polo Figueroa,
Felipe Pardo Pardo, Luis Ángel Baena, Jaime Bernal Leongómez, entre otros.

En la Universidad del Valle, el profesor Luis Ángel Baena (Cárdenas, 1999) propuso un enfoque semántico comunicativo, en el cual considera la lengua española como instrumento de comunicación, pensamiento y conocimiento basado en cuatro habilidades comunicativas (hablar, escuchar, leer y escribir).

El Ministerio de Educación Nacional (MEN), en 1987, produjo un documento de Renovación Curricular, en el cual se retoman los resultados de las investigaciones de Luis Ángel Baena. Se concibe la lengua como un instrumento de la comunicación que permite conocer, pensar y hablar de la realidad; aspectos necesarios para que el estudiante de español y literatura desarrolle su competencia comunicativa y aptitud verbal. En esta línea se asimila a competencia con capacidades comunicativas (MEN, 1987, p. 34).

Con las políticas de la Ley General de Educación de 1994 se realiza una nueva propuesta de renovación curricular en la cual se retoman, de un lado, las investigaciones sobre comunicación adelantadas en los años $80 \mathrm{y}$, por otro, se propicia un proceso de investigación, con el fin de fundamentar la constitución de nuevos lineamientos curriculares y generar un proceso de evaluación en competencias básicas, para cualificar el sistema escolar.

Con el fin de responder a las necesidades del Ministerio de Educación Nacional, la Universidad Nacional de Colombia recibió una invitación de la Secretaría de Educación del Distrito (SED) para participar en el proyecto de la evaluación de la calidad de la educación en Bogotá, como consecuencia, el equipo de investigación conformado por María Cristina Torrado, Fabio Jurado Valencia, Fernando Sarmiento Parra, entre otros, y coordinado por Manuel Vinent Solsona, aplicó una serie de evaluaciones sobre las competencias comunicativas a estudiantes entre los grados tercero y noveno, con el objeto de que las instituciones educativas reconocieran sus debilidades y fortalezas para que tomaran las medidas pertinentes.

Por otra parte, los investigadores del MEN buscan complementar la propuesta semántico comunicativa desde los aportes de la semiótica, la lingüística del texto, la pragmática y trabajos sobre cognición. De este proceso resultó la publica 
ción del documento Lineamientos Curriculares en Lengua Castellana (1998), donde se entiende al "lenguaje en términos de significación y comunicación, lo que implica una perspectiva sociocultural [...]" (MEN, 1998, p. 45).

Por la misma época, el Sistema Nacional de Pruebas del Instituto Colombiano de Fomento a la Educación Superior (SNP-ICFES) publicó, en 1998, una serie de investigación y evaluación por competencias, en la cual autores como Carlos Augusto Hernández, Alfredo Rocha de la Torre y Leonardo Verano desarrollan el concepto de competencias como posible objeto de evaluación (1998). Con estos documentos se buscó: "Convocar al país, y en especial a la comunidad educativa, a que abordemos de manera amplia y profunda [...] los exámenes de ingreso a la educación superior, partiendo de su conocimiento, análisis de la calidad [...] y de la realidad social" (Torrado, 1998).

En contraposición a las tendencias tanto del grupo de la Universidad Nacional como a los resultados de los documentos propuestos por el MEN y los lineamientos curriculares de lengua castellana respecto a la concepción de competencias, la Sociedad Colombiana de Pedagogía (Socolpe) se preocupa por las redes de significación específicas que definen la categoría competencia desde propiedades pedagógicas. De esta manera, Socolpe, con teóricos como Edgar Torres, Luis Marín, Guillermo Bustamante, Jairo Gómez y Esteban Barrantes, entre otros, buscan fundamentar el concepto de competencia desde diferentes disciplinas, tales como las ciencias del lenguaje, la filosofía y la

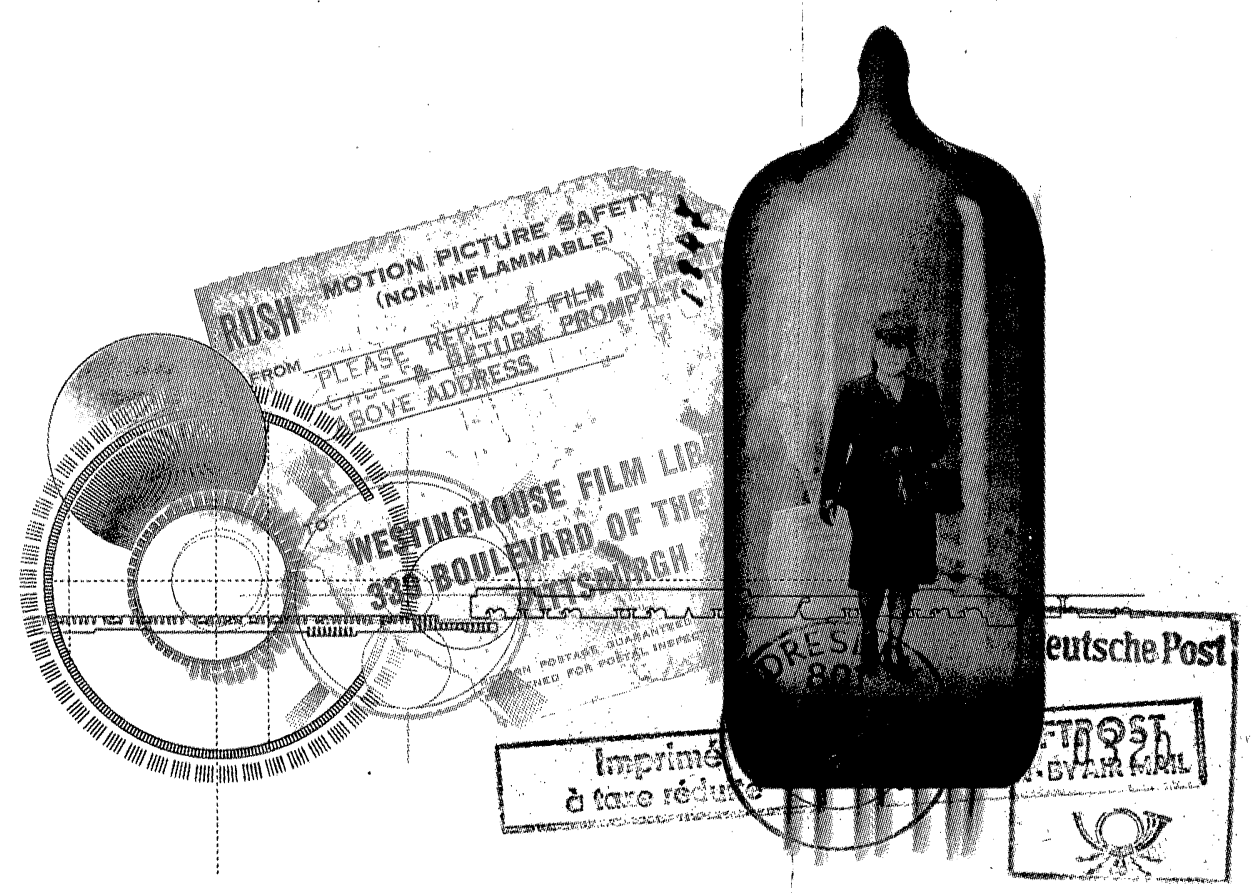

educación. Al respecto, publican tres tomos del documento "El concepto de competencia" donde profundizan tanto en una mirada interdis-ciplinar sobre las competencias, como en su recontextualización en Colombia.

De lo anterior se puede establecer que la educación Colombiana en lengua castellana ha trabajado el concepto de competencia desde tres ámbitos. En primer lugar, el teórico lingüístico, con dos variables, la teoría chomskiana, centrada en la competencia como potencialidad interna del ser humano y la de Hymes, preocupada por la relación entre competencia y actuación como capacidad humana desarrollada en contextos. En segundo lugar, desde la esfera educativa en la cual se retoman los conceptos teóricos de la lingüística para promover investigaciones con intereses pedagógicos y elaborar documentos oficiales del MEN, SNPICFES y SED. En tercer lugar, el de las concepciones que tienen los maestros de lengua castellana acerca de las competencias, que será expuesto más adelante.

\section{METODOLOGÍA}

La investigación que sustenta los resultados presentados a continuación, se abordó desde un enfoque cualitativo de tipo descriptivo, que permitió conocer las creencias, las valoraciones y los conceptos que los maestros e investigadores de educación básica, media y superior en lengua castellana tienen con respecto al concepto de competencias comunicativas.

Los sujetos entrevistados son profesores e investigadores de educación básica, media y superior, pertenecientes al sector público y privado en Bogotá. La selección obedeció a là necesidad de encontrar un grupo de maestros de educación básica y media en el área de lengua castellana que asumieran en su ejercicio docente el modelo de educación por competencias, y otro grupo de maestros e investigadores de educación superior que hayan trabajado, tanto en sus investigaciones como en sus clases, con el concepto de competencia lingüística y competencia comunicativa.

Como estrategia de recolección de información se utilizó la entrevista. La información resultante se analizó empleando la técnica de análisis de conte- 
nido, buscando distintos significados simbólicos de los testimonios para establecer un vínculo en tres niveles del lenguaje: nivel de superficie, constituido por las afirmaciones, preguntas y en general, formulaciones de los informantes; nivel analítico, ordenación de las formulaciones a través de criterios de afinidad o diferencia con los cuales se construyeron categorías para clasificar y organizar la información; y nivel interpretativo, explicación y desarrollo de cada una de las categorías encontradas dotándolas de sentido nuevo (Torrado, 1998, p. 203).

\section{RESULTADOS}

A partir del análisis de contenido de las entrevistas se determinaron cuatro categorías, seleccionadas a partir de las ideas comunes encontradas con mayor contenido significativo en las entrevistas. Tales categorías son: 1) El concepto de competencia para maestros e investigadores en lenguaje. 2) Interpretaciones y recontextualizaciones del concepto de competencia. 3) Competencias y prácticas educativas. 4) Incompatibilidad entre disposiciones gubernamentales y realidad educativa en cuanto a las competencias.

\subsection{El concepto de competencia para maestros e investigadores en lenguaje}

Los entrevistados tienen diversos conceptos de competencia, dentro de los cuales se destacan la competencia como saber hacer en contextos, la competencia como habilidad y conocimiento y la competencia como competitividad.

\subsubsection{Competencia como "saber hacer en contextos" (Vinent, 2000)}

El concepto más adoptado tanto por los maestros como por los investigadores entrevistados es el de competencia como saber hacer en contexto. Tal concepto es retomado de los investigadores de la Universidad Nacional que desarrollaron el proyecto "Hacia una cultura de la evaluación para el siglo XXI".

Las ventajas encontradas en este concepto se basan, en primer lugar, en su carácter general, en segundo lugar en la inclusión que hace tanto de los aspectos cognitivos, como de las acciones que los sujetos pueden desarrollar y en tercer lugar, el reconocimiento de la posibilidad de la aplicación del concepto en contextos (Marín, 2002) ${ }^{6}$.

\section{1.2. Concepto humanista de la competencia}

Algunos investigadores consideran que el concepto de competencia, además de un saber hacer en contexto, implica tanto la toma de conciencia de ese saber hacer, como una conciencia crítica que permita a los sujetos actuar en consecuencia con las problemáticas que se presentan en su realidad. De esta manera, la competencia desde el lenguaje, no sólo se limita a un saber hacer sino que implica la elaboración de una serie de conocimientos que permitan a los individuos desenvolverse en la vida. Es decir, competencias para saber ser humano.

\subsubsection{Competencia como conocimiento y habilidad}

En las concepciones de competencias expuestas por los entrevistados se evidencia una clara tendencia a relacionar el conocimiento y la habilidad como elementos complementarios que forman parte esencial de la competencia. Dentro de la visión de la competencia como conocimiento y habilidad encontramos varios enfoques relacionados con: la psicología cognitiva (Torrado, 2000), con los procesos de representación y construcción social del conocimiento, y con las teorías educativas constructivistas.

La concepción de competencia como conocimiento y habilidad es relacionada, por algunos entrevistados, con la teoría de las inteligencias múltiples de Howard Gardner (1985). En esta tendencia se considera que existen diversas inteligencias (lingüística, matemática, quinésica, musical, etc.) que se evidencian en las habilidades que tienen los sujetos para desempeñar diversas actividades. En tal sentido, la competencia revela las diferentes habilidades con las que cuenta el sujeto, con lo cual deja ver una determinada inteligencia.

En esta línea, algunos entrevistados consideran que la competencia implica el desarrollo de procesos cognitivos como habilidades a partir de los cuales los individuos pueden desenvolverse en

\footnotetext{
${ }^{6}$ Luis Fernando Marín argumenta que las diversas definiciones del concepto competencia, en la educación colombiana, son inseparables del concepto de contexto. En tal sentido, Marín, después de hacer una revisión de los fundamentos del concepto de competencia, identifica que los diversos usos del término son proyectados por los teóricos del MEN, ICFES y UN, hacia contextos disímiles: hacia la escuela como lugar de simulación de problemas, hacia la cotidianidad, hacia la disciplina científica, hacia el entorno cultural, hacia las situaciones nuevas o creativas y hacia la situación precisa, o determinada, resuelta idóneamente.
} 
diferentes disciplinas y adquirir cualquier tipo de conocimiento. Sin embargo, los entrevistados manifiestan un interés en los procesos cognitivos por encima de la adquisición de conocimientos específicos, puesto que la apropiada formación de los primeros da como resultado una óptima adquisición de los segundos.

Desde otra perspectiva, argumentan que las competencias son construcciones cognitivas internas que conducen a la elaboración de representaciones. En tal sentido, la competencia más que una capacidad es una representación que implica un proceso cognitivo a partir del cual los individuos logran comprender el mundo e interrelacionarse con otros individuos.

En la visión de competencia como conocimiento y habilidad, la competencia no se reduce a la constitución de procesos metales internos ni al fortalecimiento de habilidades básicas puesto que, las funciones cognitivas del individuo se proyectan hacia una educación que permita a los estudiantes una construcción social del conocimiento para responder efectivamente a las necesidades de sus contextos específicos (Hernández, Rocha y Verano, 1998, p. 14).

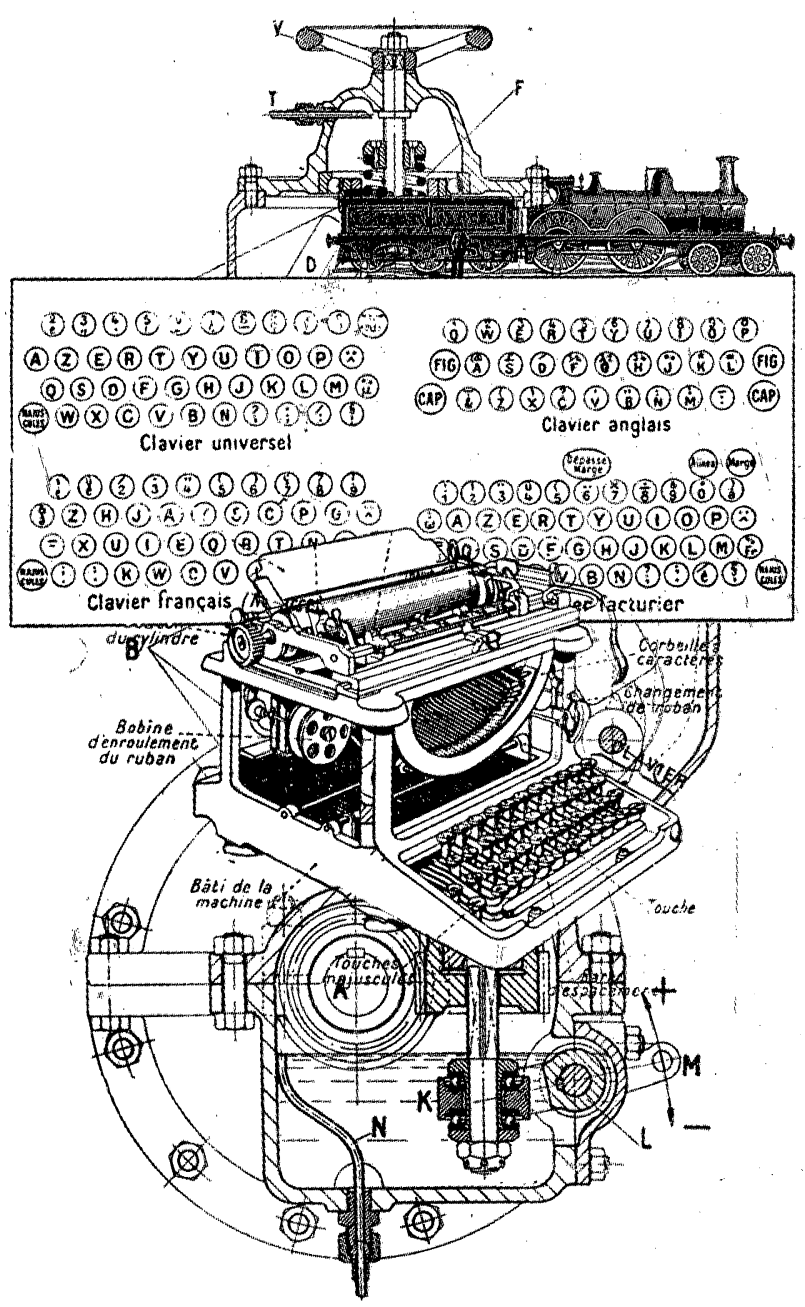

\subsubsection{Competencia y constructivismo}

Para los maestros de educación básica y media las competencias son habilidades que tienen los estudiantes para desarrollar diferentes actividades. Tales habilidades implican la consolidación de procesos mentales. En esta vía los maestros de escuela, al igual que los demás entrevistados, conceptualizan a la competencia de la misma manera que los diferentes procesos cognitivos (Piaget, 1994) que hacen hábiles a los estudiantes para responder desde y para las exigencias del medio en que se encuentran.

\subsubsection{Competencia y competitividad}

Algunos entrevistados relacionan el concepto de competencia con competitividad o competición. Con lo cual se asume que las competencias deben estar ligadas con la efectividad y con las actividades laborales ${ }^{7}$. Esta conceptualización de competencia se atribuye a las políticas neoliberales que buscan la optimización de los procesos laborales con el fin de estimular sociedades productivas donde cada sujeto esté en la capacidad de desarrollar actividades específicas dentro de un aparato productivo.

\subsection{Interpretaciones y recontextuali- zaciones del concepto de competencia}

\subsubsection{Interpretación del concepto de competencia}

Según algunos investigadores entrevistados, la mayoría de los maestros e investigadores universitarios hacen una interpretación sin rigor del concepto de competencia tomado de: Chomsky, Hymes, Verón o Cosseriu. Además, consideran que otros investigadores del campo de las competencias se limitan a interpretaciones superficiales del concepto de competencia, puesto que su interés se centra en una adaptación inmediata del término para que sea fácilmente asimilada y comprendida por los maestros de educación básica y media.

\footnotetext{
${ }^{7}$ En Australia fue organizada, en 1990, una misión gubernamental que conoció e investigó diversas experiencias de capacitación en el extranjero y declaró en su informe (COSTAC) que un enfoque de competencia para la educación y capacitación, basado en las normas dictadas por las empresas, ayudaría a abordar muchos de los problemas de la formación profesional. Posteriormente, se publicaron los lineamientos para la implantación del sistema. Tales modelos fueron propuestos para Latinoamérica durante los años noventa, por el Fondo Monetario Internacional (FMI).
} 
También consideran que el concepto de competencia como "saber hacer en un contexto particular" es fruto de una discusión superficial, que realizaron los teóricos que trabajan para el ICFES, de los postulados del discurso de la comunicación en Hymes, de la legitimidad del discurso moral en Habermas y de la interpretación de las culturas locales en Geertz. Es por ello que se han desarrollado concepciones de competencias para la educación, fruto del cambio de sentido de la teoría de la competencia comunicativa, especialmente la retomada de Hymes.

En consecuencia, algunos entrevistados piensan que es necesaria una reflexión teórica rigurosa del concepto de competencias aplicadas a la educación para que los miembros de las instituciones reguladoras de la educación y la comunidad educativa logren cualificar tanto las teorías como las prácticas del sistema educativo colombiano.

\subsubsection{Recontextualización del concepto de competencia}

Los entrevistados indican que, además de los problemas de interpretación que tuvo el concepto de competencias por parte de los investigadores en educación que construyeron los documentos de lineamientos curriculares y de evaluación por competencias, los maestros de lengua castellana en educación básica han venido realizando una continua recontextualización del término (Socolpe, 2003). Tal recontextualización representa, tanto la falta de fundamentación epistemológica, como la ausencia de reflexión y toma de conciencia con respecto a las teorías. De esta manera, algunos investigadores entrevistados argumentan que, debido a la deficiente actitud reflexiva, crítica o investigativa de los maestros de escuela, es vano tratar de implementar cualquier modelo de formación educativa, ya sea de competencia, habilidades, objetivos, logros, estándares, etc.

Con lo anterior, se evidencia que algunos investigadores entrevistados consideran que en el espacio de formación básica y media, los maestros imparten una educación irreflexiva. De esta manera, se considera al maestro como a un sujeto que adapta a sus esquemas de formación los términos que le son impuestos por el MEN y el ICFES (MEN, 1998, pp. 50-52; Universidad Nacional, 2000; Hernández, Rocha y Verano, 1998).

De manera similar, los maestros de escuela entrevistados consideran que frente a las imposi- ciones del Gobierno deben dar prioridad a los modelos de educación ya sean institucionales o personales. Los primeros han sido elaborados por el colegio en que trabajan, y los segundos han sido construidos por el maestro durante el espacio y el tiempo de su quehacer educativo. En tal sentido, para los maestros entrevistados los conceptos que provienen de las instituciones de regulación educativa son secundarios frente a los modelos de educación institucionales o personales. Es por ello que consideran que su papel epistemológico frente a las variantes disposiciones del MEN es encontrar la utilidad de los conceptos propuestos para su quehacer educativo real.

Desde esta perspectiva, el concepto de competencia, para los maestros de escuela, puede cambiar, adaptarse, resemantizarse, reinterpretarse o convertirse en otro concepto, siempre y cuando sea de utilidad para el desempeño de su trabajo en el aula de clase. Además, varios de los entrevistados indican que el trabajo por competencias en educación obedece a una moda. Puesto que, cada vez que el Ministerio de Educación Nacional impone un nuevo sistema educativo o una nueva evaluación, importa de los países desarrollados ${ }^{8}$ (MEN, 1998) un cúmulo de conceptos que los maestros e instituciones educativas deben incorporar a su modelo pedagógico.

Debido a las anteriores circunstancias histórico-políticas, los maestros de educación básica y media se han acostumbrado a una dinámica de cambio de reglas. Por lo tanto, son capaces de utilizar los conceptos sin comprometerse con ellos, puesto que más tardan los maestros en asimilar un concepto, que las instituciones reguladoras de la educación en imponer uno nuevo, sin importar que esos conceptos educativos sean incompatibles o inconmensurables con los otros.

\subsection{La competencia y las prácticas educativas}

En esta categoría describiremos la incidencia del concepto competencia en las prácticas educativas, a la luz de los testimonios dados por los maestros

\footnotetext{
${ }^{8}$ Ver: Ley 24 de 1988 que generó la creación del Sistema Nacional de Evaluación de la Calidad de la Educación en el MEN; Ley 115 de 1994: "se establecerá indicadores de logros para cada grado de los niveles educativos" (función del MEN artículo 148 Ley 115); Decreto 2343 de 1996 que establece indicativos de logros curriculares para la educación formal; Decreto 230 de 11 de febrero de 2002 que reglamenta el currículo, la evaluación y la promoción de los educandos.
} 
y los investigadores en Lengua Castellana. Desde esta perspectiva destacamos: 1) el papel de la competencia como aspecto reorientador de las prácticas pedagógicas en Colombia. 2) La competencia como concepto básico para la formación laboral de los estudiantes.

\subsubsection{La competencia como aspecto reorientador de las prácticas pedagógicas}

Dentro de las entrevistas realizadas a los maestros de educación media encontramos una marcada tendencia a valorar la competencia como un concepto que permitió modificar las prácticas pedagógicas en lengua castellana durante los últimos años de la década de los noventa. En tal sentido, los maestros encuentran que hubo cambios en los modelos pedagógicos, en la forma de relación entre el maestro y el alumno y en la forma de asumir la enseñanza del lenguaje.

\subsubsection{Modelos pedagógicos}

Para los maestros de colegio entrevistados existe una clara relación entre la teoría constructivista y la teoría de aprendizaje significativo con el modelo de formación en competencias. En esta vía, los maestros asumen que la educación ha mejorado con la asunción de las competencias dentro de las prácticas educativas. Puesto que se pasó de una educación basada en modelos memorísticos y prescriptivos (MEN, 1987) ${ }^{9}$ a una educación que privilegia la construcción del conocimiento y el aprendizaje significativo.

\subsubsection{Asunción de la lengua}

El cambio de enfoque de la educación proveniente de la renovación curricular de $1998^{10}$, además de propiciar un cambio en los modelos educativos, permitió, según los maestros entrevistados, dar un giro en la manera como asumían la enseñanza de la lengua.

Es así como de una enseñanza preocupada por normas gramaticales y ortográficas se pasa a una formación donde se privilegia la conciencia de los procesos del lenguaje y se busca la interacción comunicativa entre los estudiantes.

En consecuencia, se pasa de la enseñanza de la lengua de corte memorístico y prescriptivo al desarrollo de competencias comunicativas que permiten al alumno ser hábil para comunicarse, para argumentar, para proponer, para interpre- tar, para ser reflexivo, para ser crítico y para ser consciente de la utilidad de los procesos de comunicación en los que se ve involucrado.

\subsubsection{Ejercicio del docente}

Con base en los testimonios de los maestros entrevistados encontramos que la incidencia de las competencias en los contextos educativos, además de cambiar los modelos pedagógicos y la forma de asumir la enseñanza de la lengua por parte de los maestros, propició un cambio en la manera en que se perciben los maestros a sí mismos.

Desde esta perspectiva, los maestros dicen que pasaron de ser sujetos vigilantes del conocimiento a ser orientadores de procesos y guías de sus estudiantes. Por lo tanto, el papel del maestro cambia de un poseedor omnipotente del conocimiento a un acompañante mediador que busca con sus alumnos las herramientas más apropiadas para lograr el desarrollo de las competencias básicas necesarias para cumplir con las exigencias de las instituciones educativas.

\subsection{Incompatibilidad entre disposiciones gubernamentales y realidad educativa}

Otra gran anomalía que los entrevistados encuentran en la incidencia del término competencia en contextos educativos es la incompatibilidad que existe entre las disposiciones impuestas por los organismos gubernamentales de educación y los espacios educativos.

Algunos entrevistados argumentan que por un lado van los objetivos del MEN y por el otro va la realidad de los contextos de la educación básica y. media, puesto que los ejecutivos del MEN, en su

\footnotetext{
${ }^{9}$ Allí el cambio de un modelo memorístico de educación en lengua castellana a un modelo desde las competencias comunicativas se empieza a marcar oficialmente.

${ }^{10}$ Los lineamientos curriculares de lengua castellana publicados en 1998 buscaron un currículo autónomo articulado por el concepto de competencia, propuesto para ser aplicado en cada institución educativa desde un Proyecto Educativo Institucional (PEI) en pro del mejoramiento de la calidad educativa. Paralelamente el SNP.ICFES publicó, en 1998, la serie de "Investigación y evaluación", donde autores como Carlos Augusto Hernández, Alfredo Rocha, Leonardo Verano y María Cristina Torrado, entre otros, desarrollan el concepto de competencias como posible objeto de evaluación. Con estos documentos se buscó "Convocar al país, y en especial a la comunidad educativa, a que abordemos de manera amplia y profunda [...], los exámenes de ingreso a la educación superior, partiendo de su conocimiento, análisis de la calidad [...], y de la realidad social".
} 
gran mayoría, nunca han tenido relación directa con el ejercicio de la docencia y sus profesiones nada tienen que ver con la educación. El resultado de tal anomalía institucional es el desconocimiento o visión desatinada que el MiEN tiene del contexto escolar.

En tal sentido, los maestros se ven obligados a adaptar modelos ideales de formación en competencias a situaciones reales de uso. De esta manera, en el aula de clases hay una continua tensión entre un modelo de estudiante ideal proveniente de los textos del gobierno y un estudiante real con diversas problemáticas y posibilidades socioculturales y afectivas.

Además de la tensión entre estudiante ideal y estudiante real, y de la tensión entre disposiciones gubernamentales y realidad escolar, los investigadores entrevistados dicen que existe tanto falta de voluntad por parte de los maestros para dar solución a tales tensiones, como la falta de comprensión de los conceptos impuestos desde las políticas educativas. Por lo tanto, muchos maestros se limitan a cumplir con los horarios establecidos en sus colegios y con el desarrollo básico de sus asignaturas, independientemente del contexto y de la heterogeneidad de los alumnos con quienes trabajan.

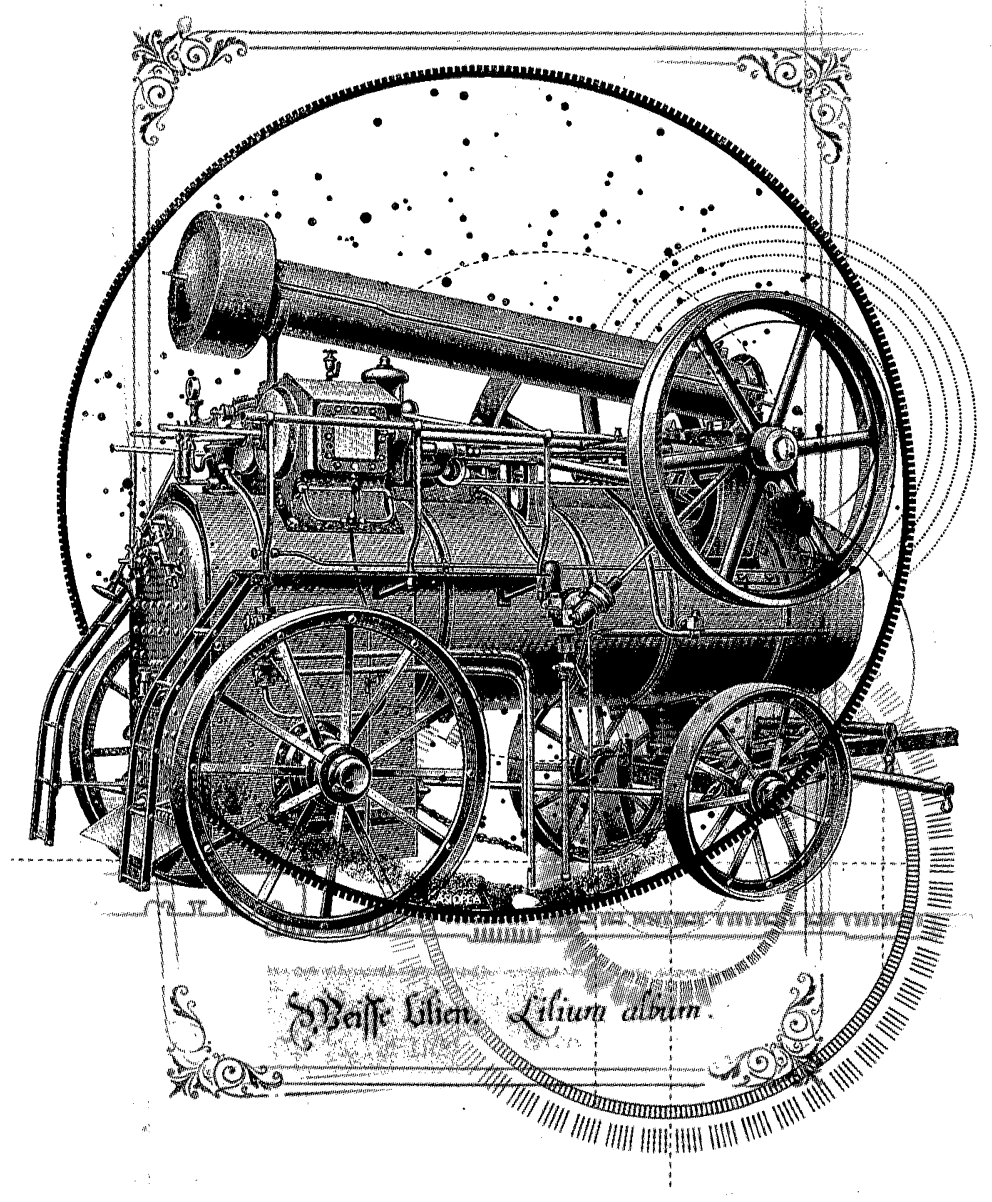

\subsection{Competencias y políticas públicas en Educación (Coraggio, 1997)}

Para los entrevistados, el modelo de formación en competencias se implementó en Colombia como una imposición de algunos países industrializados desde políticas económicas neoliberales de globalización. En tal sentido, detrás del modelo de formación en competencias están los presupuestos internacionales de desarrollo de la competitividad laboral.

Cuando las políticas internacionales de formación en competencias son traducidas a políticas nacionales se corre el riesgo, según los entrevistados, de desconocer las necesidades propias de los contextos colombianos; es por ello que se obliga a los maestros a asimilar contenidos que no les son propios, de esta manera, no se produce el impacto que, a través de las políticas educativas, los organismos gubernamentales esperan en el sistema de educación básica y media; situación que da como resultado un cuerpo profesoral interesado en cumplir con el sistema, con el fin de evitar sanciones que afecten su estabilidad laboral. En tal sentido, el continuo cambio de políticas educativas no contribuye ni a la formación, ni a la comprensión, ni a la reflexión crítica que los maestros de formación básica y media puedan hacer de cualquier concepto educativo, incluso el de competencia.

\section{A MANERA DE CONCLUSIÓN}

Las conceptualizaciones que los maestros de lengua castellana hacen del concepto de competencia están orientadas más por las necesidades socioculturales del aula de clase que por los intereses epistemológicos de competencia propuestas por Chomsky, Hymes o Habermas.

En Colombia, en el área de competencia para la lengua castellana, no existe una comunidad científica determinada que esté unida por principios, valores, creencias, métodos, técnicas, instrumentos, entre otros. Aunque podemos identificar investigadores que pertenecen a la Universidad $\mathrm{Na}-$ cional de Colombia o a Socolpe, cada uno de ellos hace un análisis y tiene una conceptualización particular del término competencia, sin que se genere una discusión científica posterior.

Las contradicciones en los documentos realizados por investigadores que han sido convocados por el MEN, la SED y el SNP-ICFES, unidas a las carencias de fundamentos epistemológicos en los maestros de educación básica y secundaria, gene- 
ran una información contradictoria y un ambiente de confusión.

En Colombia, existe una brecha entre las teorías sobre competencias educativas generadas desde el MEN y la aplicación del concepto de competencias por parte de docentes vinculados a instituciones de básica y media. En tal sentido, concluimos que no se ha elaborado un proceso de formación en el concepto de competencias por parte del gobierno y sus investigadores orientado hacia los maestros de educación básica y media.

En la medida en que los conceptos se han adaptado a las diversas circunstancias y necesidades de los contextos educativos en la educación básica y media, los maestros han asimilado, sin realizar un análisis riguroso, las competencias con el modelo constructivista y con las teorías psicológicas del aprendizaje significativo. De lo cual se deduce que los maestros han adecuado los modelos educativos, teorías y conceptos que conocen asimilándolos con el modelo educativo por competencias y, por otra parte, privilegian sus necesidades laborales, personales e institucionales frente a los modelos venidos de una realidad política-económica que les es ajena.

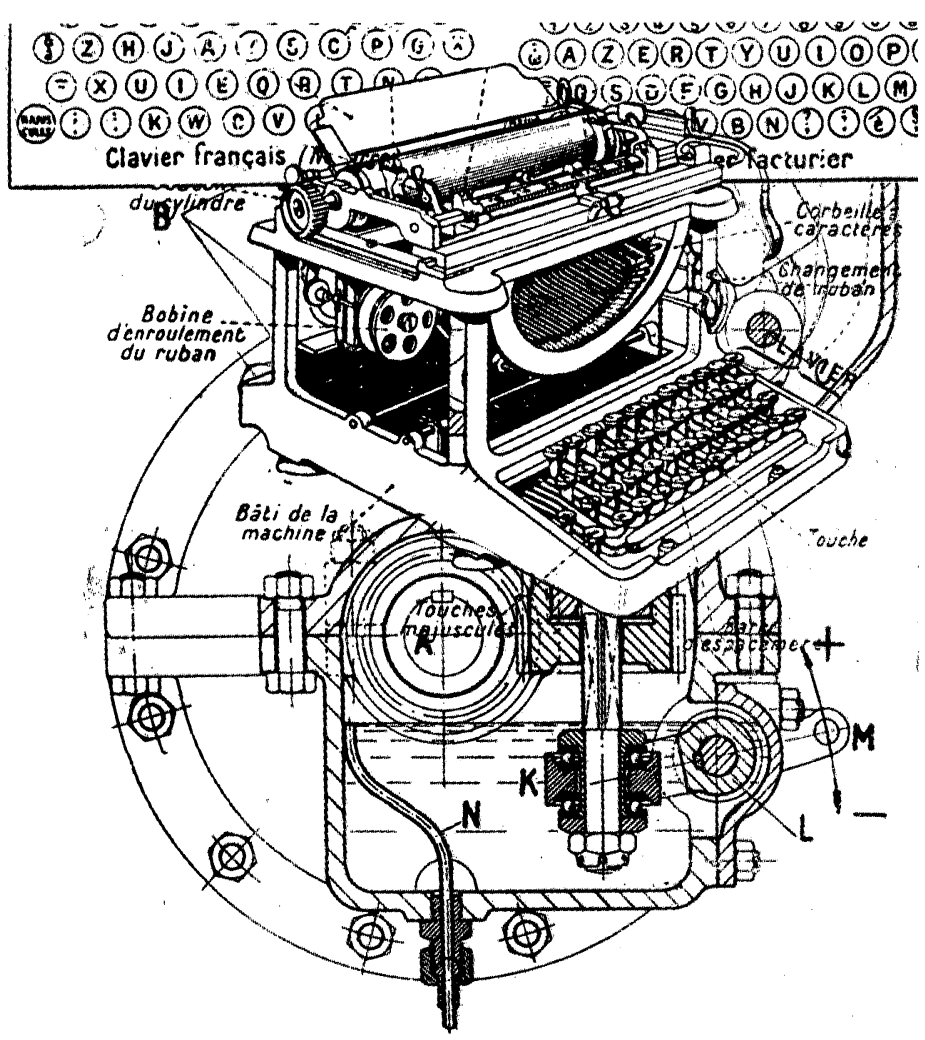

\section{BIBLIOGRAFÍA}

CÁRDENAS, Alfonso, (1999), "El aspecto semántico comunicativo: bases y proyecciones”, Enunciación, Bogotá, No. 3, Universidad Distrital Francisco José de Caldas, abril.

CHOMSKY, Noam, (1970), Aspectos de la teoría de la sintaxis, Madrid, Aguilar.

-, (1979), Reflexiones sobre el Lenguaje, Barcelona, Ariel.

CoRAGGIO, José Luis, (1997), "Economistas y educación”, en: Políticas, Instituciones y actores en educación, Buenos Aires, Novedades educativas.

DESCARTES, Renato, (1975), El discurso del método, Barcelona, Bruguera.

GARDNER, H., (1985), "Frames of Mind. The Theory of Multiple Intelligences", New York, Basic Books Inc, Publishers.

HERNÁNDEZ, Carlos Augusto, Rocha de la Torre Alfredo y Verano Leonardo, (1998), Las competencias como posible objeto de evaluación: elementos teóricos, Serie de investigación y evaluación educativa, Bogotá, SNPICFES.

HYMES, Dell, (1996), "Acerca de la competencia comunicativa”, Forma y Función, Bogotá, No. 9, Departamento de Lingüística, Universidad Nacional.

JURADO, Fabio et. al., (2000), "Hacia una cultura de la evaluación para el siglo XXI" (Taller sobre evaluación de las competencias básicas), Bogotá, Secretaría de Educación, UN de Colombia.

MARÍN, Luis Fernando, (2002), "Competencias 'saber hacer', ¿en cuál contexto?”, en: Socolpe, Bustamante Guillermo, El Concepto de Competencia II, Bogotá, Alejandría Libros.

MINISTERIO DE EDUCACIÓN NACIONAL (MEN), (1998), Lineamientos curriculares de la lengua castellana, Bogotá, Cooperativa Editorial Magisterio.

MINISTERIO DE EDUCACIÓN NACIONAL (MEN), Dirección general de capacitación y perfeccionamiento docente, (1987), Currículo y medios educativos: marcos generales de la renovación curricular de español y literatura, Bogotá, Ministerio de Educación Nacional.

MISIÓN, CIENCIA, EDUCACIÓN Y DESARROLLO, (1994), Colombia al filo de la oportunidad, Presidencia de la República - Colciencias, Bogotá.

SOCOLPE, (Sociedad Colombiana de Pedagogía), (2001), El concepto de competencia: una mirada interdisciplinar, Bogotá, tomo I, Alejandría Libros.

TORRADO, María Cristina, (1998), De la evaluación de aptitudes a la evaluación de Competencias, Bogotá, Universidad Nacional de Colombia.

—, (2000) "Educar para el desarrollo de las competencias: una propuesta para reflexionar", en: D. Bogoya et. al., Competencias y proyecto pedagógico, Bogotá, s. e.

UNIVERSIDAD NACIONAL de Colombia, (2000), Competencia y proyecto pedagógico, Bogotá, Unilibros.

VINENT, Manuel, (2000), "Un saber hacer en el ámbito de un contexto determinado", en: Secretaría de Educación, Hacia una cultura del siglo XXI (Taller sobre evaluación de las competencias básicas), Bogotá, Secretaría de Educación, Universidad Nacional de Colombia. 